\title{
Scientific Meeting 2014 "NGS Data after the Gold Rush" \& "Management Committee" Meeting, Norwich, UK
}

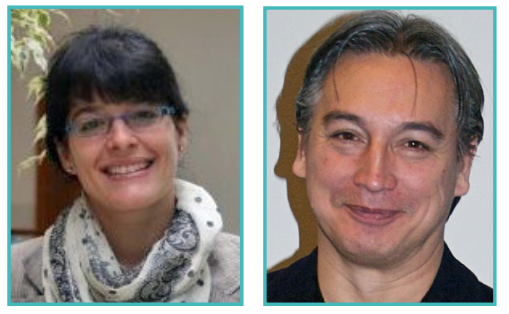

\section{Vicky Schneider', Erik Bongcam-Rudloff ${ }^{\bowtie}$}

'The Genome Analysis Centre (TGAC), Norwich, United Kingdom ${ }^{2}$ Swedish University of Agricultural Sciences, Uppsala, Sweden Schneider V and Bongcam-Rudloff E (2014) EMBnet.journal 20(Suppl A), e794. http://dx.doi.org/10.14806/ej.20.A.794

With 70 stakeholders from 28 European countries, the three-day meeting at the The Genome Analysis Centre (TGAC) facilities in Norwich (UK) explored the state-of-the-art in Next Generation Sequencing (NGS) data analysis, its current challenges and applications.

The event was opened by organiser, Dr. Vicky Schneider, who highlighted the importance of being able to host and make the most of this networking opportunity at TGAC. The first session was chaired by Dr. Ana Conesa, from the Prince Felipe Research Centre (ES), and Aleksandra Pawlik, from the Software Sustainability Institute (UK).

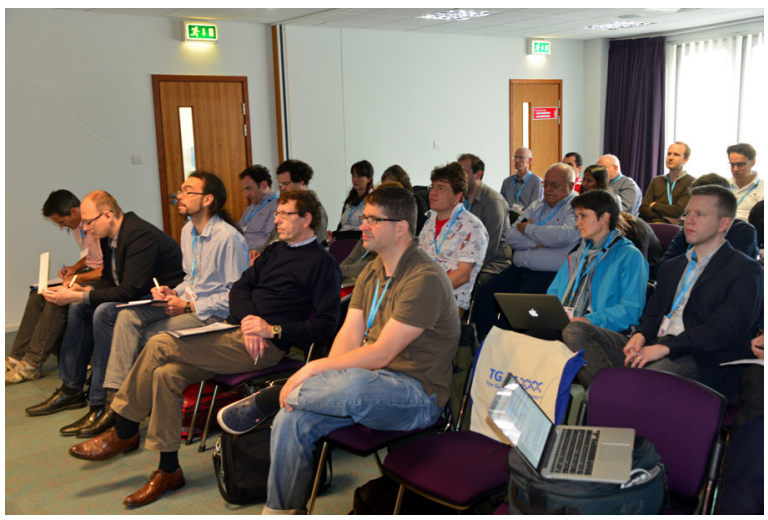

Figure 1. Audience.

During the first day of the event, participants heard presentations from leading international scientists, covering data analysis and management, bioinformatics training, functional and pathogen genomics.

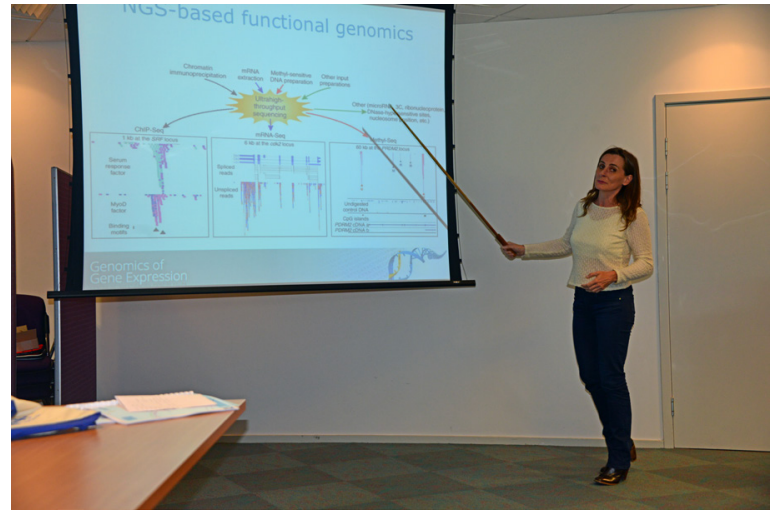

Figure 2. Ana Conesa.

The participants and speakers discussed the importance and value of the SeqAhead-COST Action' in enabling the development and coordination of a common action plan for the benefit of the scientific community, in assisting with the rise of NGS data, and in the development and optimal use of state-of-the-art bioinformatics.

The establishment of a strong European network of NGS data-analysis and informatics scientists was underlined as a key facilitator and stimulator towards the exchange of data, protocols, software, experiences and ideas.

The scientific meeting also discussed current and future directions in terms of the use of NGS technologies, further developments of highthroughput approaches, with a particular focus on parallelisation of the sequencing process, producing thousands or millions of concurrent DNA sequences. The large data volumes being generated by these new technologies require

\footnotetext{
1 seqahead.eu
} 


\section{O794}

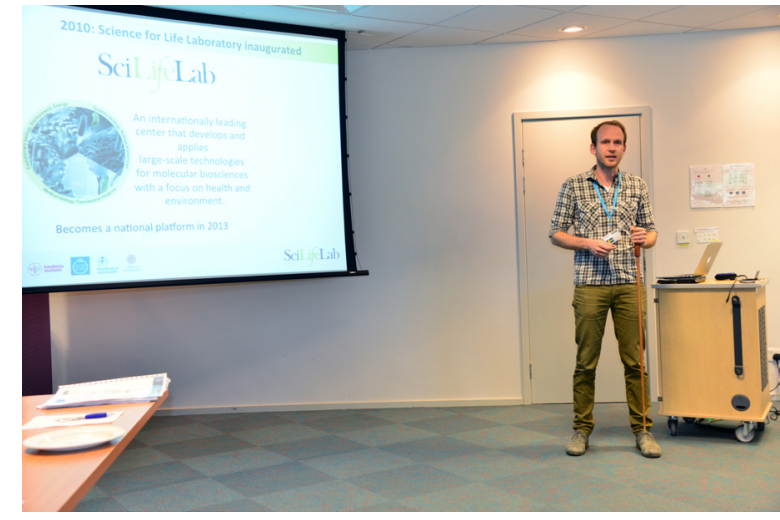

Figure 3. Ola Spjuth.

new data-handling and storage methods: solutions created and implemented by the speakers were shared and discussed, with emphasis on the critical role and need for training life scientists to make the most of existing solutions, and ensuring best practice in experimental design, in data quality control, analysis and storage, and in facilitating interoperability of data-sets and workflows.

The discussions during the scientific meeting highlighted the importance and need for opportunities to distribute knowledge and expertise via combined education and publication programs, such as the activities supported by this cost action.

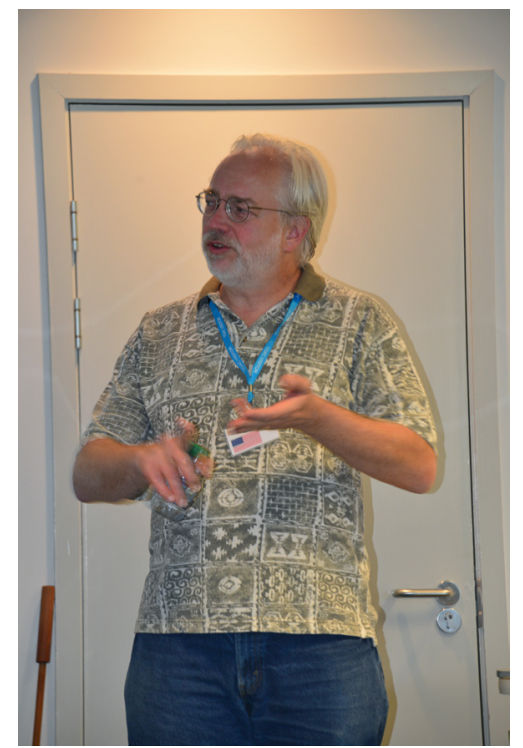

Figure 4. Dave Clements, Galaxy.
All of these activities help to unite bioinformaticians, computer scientists and biomedical scientists, harnessing their expertise to bring NGS data management and analysis to new levels of efficiency and integration.

The SeqAhead Management Committee meeting concluded the event, led by Chairman, Erik Bongcam-Rudloff, and Vice-Chair, Terri Attwood

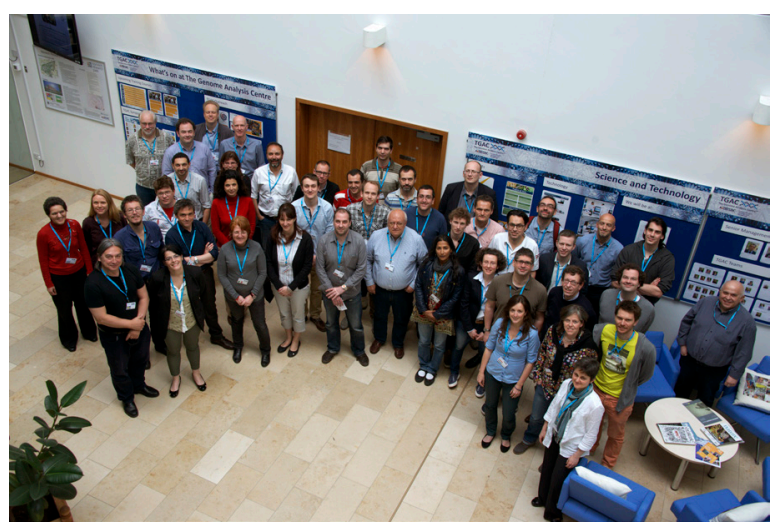

Figure 5. Group picture.

Speakers were happy to share their presentations, which can be found at: http://www.tgac. ac.uk/SegAhead Scientific Meeting and Management Committee Meeting/

Some voices from the event:

Matt Clark, Plant and Microbial Genomics Group Leader, said, "NGS is a large, growing and rapidly moving field, it's critical to keep your eye on the latest technical breakthroughs and best practices both in the lab and in bioinformatics to do the best possible research. Meetings such as this are critical to sharing knowledge across the EU and to ensure we learn from each other rapidly."

Terri Atwood, Professor of Bioinformatics at the University of Manchester and Vice-Chair of SeqAhead, said: "Networks like SeqAhead are vital both for allowing scientists to share best practice in the development and use of cuttingedge research tools, and for helping to train the 'next generation' of next generation sequencing researchers!" 


\section{4}

\section{Chair's and Conference Committees}

\section{Scientific Organisers}

Vicky Schneider, The Genome Analysis Centre (TGAC), UK

Erik Bongcam-Rudloff, Swedish University of Agricultural Sciences in Uppsala, Sweden

Ralf Herwig, Max Planck Institute for Molecular Genetics, Germany

Thomas Svensson, Karolinska Institute, Sweden

Andreas Gisel, Institute for Biomedical Tecnologies, Italy

Ana Conesa, Prince Felipe Research Centre, Spain

Eija Korpelainen, CSC, Finland

Steve Pettiffer, University of Manchester, UK

Veli Makinen, University of Helsinki, Finland

Alberto Policriti, University of Udine, Italy

Gert Vriend, Netherlands Bioinformatics Centre, Netherlands

Jacques van Helden, University of Brussels, Belgium

\section{Event Organiser}

Matt Drew, The Genome Analysis Centre (TGAC), UK

\section{Scientific Committee}

Vicky Schneider, The Genome Analysis Centre (TGAC), UK

Jean Imbert, Technological Advances for Genomics and Clinics, France

Pall Melsted, University of Iceland

Pasha Baranov, University College Cork, Ireland

Ana Conesa, Prince Felipe Research Centre, Spain

Pavlos Antoniou, Cyprus Insistute of Neurology and Genetics, Cyprus

Sophia Kossida, Biomedical Research Foundation of the Academy of Athens, Greece

Jose Valverde, CNB/CSIC, Spain

Endre Barta, Agricultural Biotechnology Centre, Hungary

Antonio Marco, University of Essex, UK

Rute Fonseca, University of Copenhagen, Denmark

Claude Muller, Institute of Immunology, Luxembourg

Manolis Christodoulakis, University of Cyprus 